\title{
BILIPSCHITZ EQUIVALENCE OF TREES AND HYPERBOLIC FILLINGS
}

\author{
JEFF LINDQUIST
}

\begin{abstract}
We show that quasi-isometries between uniformly discrete bounded geometry spaces that satisfy linear isoperimetric inequalities are within bounded distance to bilipschitz equivalences. We apply this result to regularly branching trees and hyperbolic fillings of compact, Ahlfors regular metric spaces.
\end{abstract}

\section{INTRODUCTION}

In this note we combine results from Wh with results from MPR both to generalize a theorem of Papasoglu $\mathrm{Pa}$ and to prove that the vertex sets of hyperbolic fillings of quasisymmetric, compact, Ahlfors regular metric spaces are bilipschitz equivalent. In $\mathrm{Pa}$, Papasoglu proves that (the vertices of) $k$-ary homogeneous trees are bilipschitz equivalent whenever $k \geq 3$. A map between metric spaces $f:\left(X, d_{X}\right) \rightarrow\left(Y, d_{Y}\right)$ is a bilipschitz equivalence if it is a bijection and there exists a constant $C>0$ such that for all $x, x^{\prime} \in X$ we have

$$
\frac{1}{C} d_{X}\left(x, x^{\prime}\right) \leq d_{Y}\left(f(x), f\left(x^{\prime}\right)\right) \leq C d_{X}\left(x, x^{\prime}\right) .
$$

To view a connected graph $X=\left(V_{X}, E_{X}\right)$ as a metric space, we use the graph metric. This means each edge of $X$ is taken to be isometric to an interval of length 1. For $x, x^{\prime} \in V_{X}$, it follows that the quantity $d_{X}\left(x, x^{\prime}\right)$ is the fewest number of edges required to connect $x$ to $x^{\prime}$.

Bilipschitz equivalence is a strong property that is not immediate in many situations. One has the weaker notion of a quasi-isometry which is a map that is bilipschitz at large scales. More formally, a map between metric spaces $f:\left(X, d_{X}\right) \rightarrow$ $\left(Y, d_{Y}\right)$ is a quasi-isometry if there exist constants $C, D>0$ such that for all $x, x^{\prime} \in X$ we have

$$
\frac{1}{C} d_{X}\left(x, x^{\prime}\right)-D \leq d_{Y}\left(f(x), f\left(x^{\prime}\right)\right) \leq C d_{X}\left(x, x^{\prime}\right)+D
$$

and such that every point in $Y$ is within distance $C$ of $f(X)$. Note that a bilipschitz equivalence is a bijective quasi-isometry with constant $D=0$. A natural question to ask is whether a quasi-isometry can be promoted to a bilipschitz equivalence under the right conditions. A positive answer is given by Whyte [Wh] who showed that a quasi-isometry between $U D B G$ spaces is within bounded distance from a

Received by the editors August 3, 2017, and, in revised form, September 3, 2017, February 7, 2018, and June 26, 2018.

2010 Mathematics Subject Classification. Primary 30C65; Secondary 52C99, 05C63.

At the University of Helsinki, the author was supported by Academy of Finland grants 297258 and 308759. At the University of California, Los Angeles, the author was partially supported by NSF grants DMS-1506099 and DMS-1162471.

This work was based on work from the author's thesis. 
bilipschitz equivalence if a certain homological condition holds. Here a $U D B G$ space is a metric space that is uniformly discrete with bounded geometry. A metric space $(Z, d)$ is uniformly discrete if there is a constant $c>0$ such that for all $z, z^{\prime} \in Z$ with $z \neq z^{\prime}$ we have $d\left(z, z^{\prime}\right)>c$. A metric space $(Z, d)$ is said to have bounded geometry if it is uniformly discrete and if for all $r>0$ there is a constant $N_{r}>0$ such that for all $z \in Z$ we have $|B(z, r)| \leq N_{r}$. Here and elsewhere, if $A$ is a set, then $|A|$ denotes the cardinality of $A$. Also, $B(z, r)$ is the open ball of radius $r$ centered at $z$.

Whyte's results involve boundary estimates which are reminiscent of linear isoperimetric inequalities. Let $X=\left(V_{X}, E_{X}\right)$ be a graph. For a subset $A \subseteq V_{X}$, we define the boundary of $A$ as $\partial A=\left\{x \in V_{X}: x \notin A\right.$ and $\left.d(x, A) \leq 1\right\}$. To say $X$ (or $V_{X}$ ) satisfies a linear isoperimetric inequality means that there is a constant $C>0$ such that for all finite $A \subseteq V_{X}$ we have $|A| \leq C|\partial A|$.

For graphs $X=\left(V_{X}, E_{X}\right)$, we will always view the spaces $V_{X}$ as metric spaces with the restriction of the graph metric. Our conditions on $X$ will imply that the spaces $V_{X}$ are $U D B G$ spaces. This allows us to apply the results in [Wh to prove the following theorem.

Theorem 1.1. Let $X$ and $Y$ be connected graphs with their graph metrics. Suppose $X$ and $Y$ are quasi-isometric, have bounded degree, and satisfy linear isoperimetric inequalities. Then, the vertex sets $V_{X}$ and $V_{Y}$ are bilipschitz equivalent. Moreover, if $f: X \rightarrow Y$ is a quasi-isometry, then there is a bilipschitz equivalence within bounded distance of $\left.f\right|_{V_{X}}$.

When referring to distance between functions, we use the sup norm. This means that $f, g: X \rightarrow Y$ are within bounded distance if $\sup _{x \in X} d_{Y}(f(x), g(x))<\infty$. In Theorem 1.1, the distance between $f$ and the bilipschitz equivalence can be bounded by a quantity that depends on the data of the theorem statement. By data we mean the quasi-isometry constants, the degrees of $X$ and $Y$, and the isoperimetric inequality constants of $X$ and $Y$. A proof of this dependency involves following the proofs of both [Wh, Theorem 4.1] and [Wh, Theorem 7.6] with the constants used in the proof of Theorem 1.1 and will be omitted.

Theorem 1.1 allows us to generalize Papasoglu's result to more exotic trees that satisfy linear isoperimetric inequalities. We call these trees essentially pseudoregular trees. Such trees were studied in the work of Martínez-Pérez and Rodríguez [MPR]. Their results, together with a quasisymmetric characterization from [DS97, yield the following corollary.

Corollary 1.2. Let $X$ and $Y$ be essentially pseudo-regular trees with the graph metric. Then, $V_{X}$ and $V_{Y}$ are bilipschitz equivalent.

An essentially pseudo-regular tree is a rooted, visual tree with bounded degree with a pseudo-regular maximal geodesically complete subtree. A tree is rooted if it has a specified "root" vertex, pseudo-regular if it branches regularly, visual if it does not have arbitrarily long "dead ends", and of bounded degree if there is a uniform bound on the number of edges connecting to any particular vertex. For precise definitions we refer the reader to Section 2.

The class of trees in Corollary 1.2 is larger than what is considered in [Pa]. For example, given any $M \geq 2$ and any sequence $\left(g_{i}\right)_{i \in \mathbb{N}}$ with $2 \leq g_{i} \leq M$ for each $i$, there is a unique rooted tree with root $O$ such that every vertex on level $n$ has $g_{n+1}$ children (here the level of a vertex refers to its distance to $O$ ). In this example, 
$g_{i} \geq 2$ guarantees that the tree is pseudo-regular and $g_{i} \leq M$ guarantees that the tree has bounded degree. Every vertex having children guarantees that the tree is visual. The $k$-regular tree $(k \geq 3)$ corresponds to $g_{1}=k$ and $g_{n}=k-1$ for $n \geq 2$.

For more generality, one can perform a construction where the number of children each vertex has is individually controlled (and even have some with 1 or 0 children). In these constructions one must be careful to remain in the class of essentially pseudo-regular trees.

A metric space $(X, d)$ is Ahlfors $Q$-regular if, for $\mu$ the Hausdorff $Q$ measure induced by $d$, there are constants $c, C>0$ such that for all $0<r \leq \operatorname{diam}(X)$ we have $c r^{Q} \leq \mu(B(x, r)) \leq C r^{Q}$. Theorem 1.1 has another corollary when one considers hyperbolic fillings of compact, Ahlfors regular metric spaces.

Corollary 1.3. Let $\left(Z, d_{Z}\right)$ and $\left(W, d_{W}\right)$ be quasisymmetrically equivalent, compact, Ahlfors regular metric spaces. Let $X=\left(V_{X}, E_{X}\right)$ and $Y=\left(V_{Y}, E_{Y}\right)$ be hyperbolic fillings of $Z$ and $W$. Then, $V_{X}$ and $V_{Y}$ are bilipschitz equivalent.

A homeomorphism $f:\left(X, d_{X}\right) \rightarrow\left(Y, d_{Y}\right)$ between metric spaces is a quasisymmetry if there exists a homeomorphism $\eta:[0, \infty) \rightarrow[0, \infty)$ such that for all distinct $x, y, z \in X$, we have

$$
\frac{d_{Y}(f(x), f(y))}{d_{Y}(f(x), f(z))} \leq \eta\left(\frac{d_{X}(x, y)}{d_{X}(x, z)}\right) .
$$

Two metric spaces are quasisymmetrically equivalent if there is a quasisymmetry from one onto the other. The identity map from a space to itself is a quasisymmetry, so as a special case of Corollary 1.3 we see that any two hyperbolic fillings of a given compact, Ahlfors regular metric space are bilipschitz equivalent.

Hyperbolic fillings are graph approximations of metric spaces formed by covering the metric space with specific balls and connecting two balls with an edge if they overlap. For precise constructions, we refer the reader to $[\mathrm{BuS}],[\mathrm{BP}]$, and [Lin16]. It follows from the work in [MPR that hyperbolic fillings of these spaces satisfy a linear isoperimetric inequality and thus quasi-isometries may be promoted to bilipschitz equivalences in this setting. As fillings of quasisymmetric spaces are quasi-isometric, the corollary follows.

Section 2 contains definitions and preliminaries for the rest of the paper. In Section 3 we state the relevant results from Wh and MPR. In Section 4 we prove the results stated in the introduction, namely Theorem 1.1 and its two corollaries.

\section{DeFinitions AND PRELIMINARIES}

Here we make our definitions precise.

We wish to impose a condition on trees that forces regular branching. In MPR there is such a condition which they call pseudo-regularity. To fully define this we need some notation, also borrowed from [MPR. Given a tree $T$ and points $x, y \in T$, we let $[x y]$ denote the (unique) geodesic connecting $x$ to $y$. For a fixed point $v \in T$ and any point $x \in T$, we define

$$
T_{x}^{v}=\{y \in T: x \in[v y]\} .
$$

Heuristically, $T_{x}^{v}$ is the subtree of $T$ starting at $x$ that grows away from $v$.

In the following, $S\left(v_{0}, t\right)$ is the sphere of radius $t$ centered at $v_{0}$ in the graph metric. 
Definition 2.1. The degree of a vertex $v$ in a graph is the number of edges $e$ emanating from $v$, which we write as $\operatorname{deg}(v)$. A graph has bounded degree if there is a constant $\mu>0$ such that for all $v \in V$ we have $\operatorname{deg}(v) \leq \mu$.

A rooted graph is a graph with a distinguished vertex $v_{0}$. If the graph is also a tree, we call it a rooted tree. A rooted graph $T$ is visual if there is a constant $C>0$ such that for every vertex $v \in V$, there is an infinite geodesic ray $I$ with endpoint $v_{0}$ such that $d(v, I) \leq C$. In the language of [MPR], this means $v_{0}$ is a pole of $T$.

A rooted tree $\left(T, v_{0}\right)$ is geodesically complete if whenever $f:[0, t] \rightarrow T$ is an isometric embedding with $f(0)=v_{0}$, there is an isometric embedding $F:[0, \infty) \rightarrow$ $T$ such that $f(s)=F(s)$ for all $s \in[0, t]$. Given a rooted tree $\left(T, v_{0}\right)$, we define $\left(T_{\infty}, v_{0}\right)$ as the unique geodesically complete subtree with the same root $v_{0}$ that is maximal under inclusion. That a unique maximal geodesically complete subtree exists follows from Zorn's Lemma; see [MPM, Theorem 10.1].

Given a rooted tree $\left(T, v_{0}\right)$ and $K>0$ we say $\left(T, v_{0}\right)$ is $K$-pseudo-regular if for every $t \in \mathbb{N}$ and every $a \in S\left(v_{0}, t\right)$, there exist at least two points in $S\left(v_{0}, t+K\right) \cap T_{a}^{v_{0}}$. We say $\left(T, v_{0}\right)$ is pseudo-regular if it is $K$-pseudo-regular for some $K$. It is easy to see that pseudo-regular trees are geodesically complete. Intuitively, a $K$-pseudoregular tree branches off from any infinite geodesic based at root $v_{0}$ at least once every $K$ levels. This leads to the bound $|B(a, N K)| \geq\left(2^{N}-1\right) K$ for any vertex $a$ in a $K$-pseudo-regular tree, where $|B(a, N K)|$ denotes the number of vertices in the $N K$ ball centered at $a$. A rooted tree is essentially pseudo-regular if it is visual, has bounded degree, and the maximal geodesically complete subtree is pseudo-regular.

Let $X=(V, E)$ be a connected graph. The combinatorial Cheeger isoperimetric constant of $X$ is $h(X)=\inf _{A}|\partial A| /|A|$ where $A$ ranges over nonempty finite subsets of $V$; this constant quantifies the existence of an isoperimetric inequality in $X$. In particular, $X$ satisfies a linear isoperimetric inequality if and only if $h(X)>0$.

Remark 2.2. We note that if $\left(T, v_{0}\right)$ is visual, then from [MPR, Proposition 3.8] it follows that there is a quasi-isometry $\left(T, v_{0}\right) \rightarrow\left(T_{\infty}, v_{0}\right)$.

We will use the end space definition from [MPR] of the boundary at infinity of a tree. For this, let $\left(T, v_{0}\right)$ be a rooted tree.

Definition 2.3. The end space of the rooted tree $\left(T, v_{0}\right)$ is

$$
\operatorname{end}\left(T, v_{0}\right)=\left\{F:[0, \infty) \rightarrow T \mid F(0)=v_{0} \text { and } F \text { is an isometric embedding }\right\} .
$$

We define the Gromov product at infinity of two elements $F, F^{\prime} \in \operatorname{end}\left(T, v_{0}\right)$ as

$$
\left(F \mid F^{\prime}\right)_{v_{0}}=\sup \left\{t \geq 0: F(t)=F^{\prime}(t)\right\} .
$$

We then define a metric $d=d_{v_{0}}$ on $\operatorname{end}\left(T, v_{0}\right)$ by $d\left(F, F^{\prime}\right)=e^{-\left(F \mid F^{\prime}\right)_{v_{0}}}$. Here $d$ is actually an ultrametric. This means if $F, G, H \in \operatorname{end}\left(T, v_{0}\right)$, then $d(F, H) \leq$ $\max (d(F, G), d(G, H))$. We often write $\partial_{\infty} T$ for $\operatorname{end}\left(T, v_{0}\right)$.

In [DS97, there is a characterization of metric spaces that are quasisymmetrically equivalent to the standard $1 / 3$ Cantor set, denoted here as $C_{1 / 3}$.

Theorem 2.4 ([DS97, Theorem 15.11]). A compact metric space $(X, d)$ is quasisymmetrically equivalent to $C_{1 / 3}$ if it is bounded, complete, doubling, uniformly perfect, and uniformly disconnected.

For convenience, we recall some of the terminology. Let $(X, d)$ be a metric space. 
From [DS97, Definition 15.1], $X$ is uniformly disconnected if there is a constant $C>0$ such that for each $x \in X$ and $r>0$ there is a closed subset $A \subseteq X$ with $B(x, r / C) \subseteq A \subseteq B(x, r)$ and $d(A, X \backslash A) \geq r / C$. Ultrametric spaces are uniformly disconnected by [DS97, Proposition 15.7].

$X$ is uniformly perfect if there are constants $C>1$ and $R>0$ such that for every $x \in X$ and $0<r<R$ there is an $x^{\prime} \in X$ with $r / C<d\left(x, x^{\prime}\right) \leq r$.

$X$ is doubling if there exists a constant $N>0$ such that every ball $B(x, r) \subseteq X$ can be covered by at most $N$ balls of radius $r / 2$.

Complete, bounded, doubling metric spaces are compact as bounded, doubling metric spaces are totally bounded.

For Corollary 1.2 we are concerned with essentially pseudo-regular trees. By Remark 2.2, these trees are quasi-isometric to pseudo-regular trees with bounded degree. We now prove that the end spaces of pseudo-regular trees with bounded degree satisfy the criteria in Theorem 2.4 .

Lemma 2.5. Let $\left(T, v_{0}\right)$ be a pseudo-regular tree with bounded degree. Then, $\partial_{\infty} T$ is quasisymmetrically equivalent to $C_{1 / 3}$.

Proof. With [DS97, Theorem 15.11] it suffices to show $\partial_{\infty} T$ is bounded, complete, doubling, uniformly perfect, and uniformly disconnected.

From [MPR, Proposition 3.3] $\partial_{\infty} T$ is a complete, bounded ultrametric space. Thus, $\partial_{\infty} T$ is also uniformly disconnected by [DS97, Proposition 15.7].

The fact that $\partial_{\infty} T$ is uniformly perfect follows from the fact that $\left(T, v_{0}\right)$ is pseudo-regular. This is proven in [MPR, Proposition 3.20].

We show $\partial_{\infty} T$ is doubling. Let $B(F, r)$ be a ball in $\partial_{\infty} T$. Let

$$
M=\sup \{m: F(k)=G(k) \text { for all } G \in B(F, r), k \leq m\} .
$$

If $M=\infty$, then $B(F, r)$ consists of a single point, so in this case it can be covered by one ball of radius $r / 2$. Otherwise, $r \geq e^{-(M+1)}$ and so $r / 2>e^{-(M+2)}$. Let $\operatorname{deg}(v) \leq \mu$ for $v \in V$. Hence, if $G \in B(F, r)$, then $G(M)=F(M)$ and there are at most $\mu^{2}$ possibilities for $G(M+2)$. If $G, H \in B(F, r)$ are such that $G(M+2)=$ $H(M+2)$, then $d(G, H) \leq e^{-(M+2)}<r / 2$. It follows that $B(F, r)$ is contained in $\mu^{2}$ balls of radius $r / 2$.

The quasisymmetries induced on the end spaces of pseudo-regular trees with bounded degree give rise to quasi-isometries of essentially pseudo-regular trees.

Lemma 2.6. Let $\left(T, v_{0}\right)$ and $\left(U, w_{0}\right)$ be essentially pseudo-regular trees. Then $T$ and $U$ are quasi-isometric.

It is important that essentially pseudo-regular trees are visual so as to apply Remark 2.2. The construction of a quasi-isometry between $T_{\infty}$ and $U_{\infty}$ is given by [BuS, Theorem 7.2.1]; we provide the main idea of the construction here.

Sketch of proof of Lemma 2.6. By Remark 2.2 it suffices to construct a quasi-isometry $f$ between $\left(T_{\infty}, v_{0}\right)$ and $\left(U_{\infty}, w_{0}\right)$. By Lemma 2.5, there is a quasisymmetry $\varphi: \partial_{\infty} T_{\infty} \rightarrow \partial_{\infty} U_{\infty}$. For $v \in T_{\infty}$ set

$$
B_{v}=\left\{F \in \partial_{\infty} T_{\infty}: v \in F([0, \infty))\right\}
$$

and likewise define $B_{w}$ for $w \in U_{\infty}$. Define $f(v)=w$ where $w \in U_{\infty}$ is a vertex of maximal distance from $w_{0}$ such that $\varphi\left(B_{v}\right) \subseteq B_{w}$ (such a vertex exists as $B_{w_{0}}=$ $\left.\partial_{\infty} U_{\infty}\right)$. We then show there is a constant $C>0$ such that if $v, v^{\prime} \in T_{\infty}$ with 
$\left|v-v^{\prime}\right| \leq 1$, then $\left|f(v)-f\left(v^{\prime}\right)\right| \leq C$. From our tree structure we may assume without loss of generality that $B_{v^{\prime}} \subseteq B_{v}$. Let $w=f(v)$ and $w^{\prime}=f\left(v^{\prime}\right)$. We conclude that $B_{w^{\prime}} \subseteq B_{w}$ and, by using a common point and the quasisymmetry condition, that there is a uniform bound on $\left|w-w^{\prime}\right|$. Constructing $g:\left(U_{\infty}, w_{0}\right) \rightarrow\left(T_{\infty}, v_{0}\right)$ similarly and checking that $f$ and $g$ are coarse inverses of one another completes the proof.

We will also work with some homological terminology as in [Wh]. In the remainder of this section we define what is needed.

Given a graph $X=(V, E)$ with bounded degree, we define a 0 -chain $\mathbf{c}$ to be a function $\mathbf{c}: V \rightarrow \mathbb{Z}$ and a 1 -chain $\mathbf{b}$ to be a function $\mathbf{b}: E \rightarrow \mathbb{Z}$. We represent 0 -chains as formal sums $\mathbf{c}=\sum_{v \in V} c_{v} v$ with $c_{v}=\mathbf{c}(v)$ and 1-chains as formal sums $\mathbf{b}=\sum_{e \in E} b_{e} e$ with $b_{e}=\mathbf{b}(e)$. We call a chain bounded if its coefficients are bounded. Let $C_{0}^{b}(X)$ denote the set of bounded 0-chains and $C_{1}^{b}(X)$ the set of bounded 1-chains. Given an orientation on $E$, meaning we view each edge as an ordered pair $e=\left(e_{+}, e_{-}\right)$, we define the boundary map $\partial: C_{1}^{b}(X) \rightarrow C_{0}^{b}(X)$ by defining $\partial e=e_{+}-e_{-}$and extending linearly. The orientation on $E$ is only used in this paper to define $\partial$.

We now define uniformly finite homology for $U D B G$ spaces. This is the tool used in Wh. Let $Z$ be a $U D B G$ space. For $r>0$, define a graph $X_{r}=\left(V_{r}, E_{r}\right)$ where $V_{r}=Z$ and

$$
E_{r}=\left\{\left(z, z^{\prime}\right): z, z^{\prime} \in Z, 0<d\left(z, z^{\prime}\right) \leq r\right\} .
$$

This is the 1-dimensional subcomplex of the $r$-Rips complex as defined in [Wh]. The uniformly finite homology of $Z$ is defined as a limit of the homology formed from $X_{r}$ as $r \rightarrow \infty$ and the sets of chains are denoted $C_{0}^{u f}(Z)$ and $C_{1}^{u f}(Z)$. This means $C_{0}^{u f}(Z)=C_{0}^{b}\left(X_{1}\right)$ and $C_{1}^{u f}(Z)=\bigcup_{r>0} C_{1}^{b}\left(X_{r}\right)$.

Given $U D B G$ spaces $Z$ and $W$, a chain $\mathbf{c} \in C_{0}^{u f}(Z)$, and a quasi-isometry $f: Z \rightarrow W$, we define $f_{*}(\mathbf{c}) \in C_{0}^{u f}(W)$ as follows. To each $z \in Z$, associate the 0 -chain $\mathbf{z}=1 \cdot z$. We define $f_{*}(\mathbf{z}):=1 \cdot f(z) \in C_{0}^{u f}(W)$. We then extend linearly, so if $c=\sum_{z \in Z} c_{z} \mathbf{z}$ we have

$$
f_{*}(\mathbf{c})=\sum_{z \in Z} c_{z} f_{*}(\mathbf{z})
$$

We see that $f_{*}(\mathbf{c}) \in C_{0}^{u f}(W)$ as this can be written

$$
f_{*}(\mathbf{c})=\sum_{w \in W}\left(\sum_{\{z \mid f(z)=w\}} c_{z}\right) w
$$

and, as $f$ is a quasi-isometry and $Z$ is a $U D B G$ space, there is a uniform bound on $|\{z \mid f(z)=w\}|$.

We define an equivalence relation on $C_{0}^{u f}(Z)$ by setting $\mathbf{c} \sim \mathbf{c}^{\prime}$ if and only if there exists $\mathbf{b} \in C_{1}^{u f}(Z)$ such that $\partial \mathbf{b}=\mathbf{c}-\mathbf{c}^{\prime}$. We let $[\mathbf{c}]$ denote the equivalence class of $\mathbf{c}$ under this relation. Let $\mathbf{Z}=\sum_{z \in Z} z$. We call $[\mathbf{Z}]$ the fundamental class of $Z$.

Remark 2.7. One reason Whyte uses the Rips complex is that he has no graph structure. In our situation (specifically in a graph with bounded degree), one obtains equivalent homology from the equivalence $\mathbf{c} \sim \mathbf{c}^{\prime}$ if and only if there exists $\mathbf{b} \in C_{1}^{b}(X)$ such that $\partial \mathbf{b}=\mathbf{c}-\mathbf{c}^{\prime}$. 
For the graphs $X=\left(V_{X}, E_{X}\right)$ that we consider, the spaces $V_{X}$ are $U D B G$ spaces. Uniform discreteness follows from using the graph metric, while the bounded geometry condition follows as our graphs have bounded degree.

\section{Results from Whyte and Martínez-PÉrez and Rodríguez}

Here we state the results from $[\mathrm{Wh}]$ and $[\mathrm{MPR}]$ relevant for our setting. We start with Whyte's criteria for promotion of a quasi-isometry to a bilipschitz equivalence.

Theorem 3.1 ( $[\mathrm{Wh}$, Theorem 4.1]). Let $f: Z \rightarrow W$ be a quasi-isometry between $U D B G$ spaces with $f_{*}(\mathbf{Z}) \sim \mathbf{W}$. Then, there is a bilipschitz map at bounded distance from $f$.

To apply this, we need a condition that implies $\left[f_{*}(\mathbf{Z})-\mathbf{W}\right]=[\mathbf{0}]$, where $[\mathbf{0}]$ is the equivalence class corresponding to the 0 -chain with all coefficients 0 . This is achieved using an isoperimetric inequality and Theorem 3.2. Given a $U D B G$ metric space $(Z, d)$, a subset $S \subseteq Z$, and $r>0$, we define the $r$-boundary of $S$ as the set $\partial_{r}(S)=\{z \in Z: z \notin S$ and $d(z, S) \leq r\}$. Note that for vertex sets of graphs with distances induced from the graph metric, if $A$ is a set of vertices, then $\partial_{1} A=\partial A$. Given a 0 -chain $\mathbf{c}=\sum c_{z} z$ and a finite subset $S \subseteq Z$, we write

$$
\sum_{S} \mathbf{c}=\sum_{s \in S} c_{s}
$$

Theorem 3.2 (【Wh, Theorem 7.6]). Let $Z$ be a $U D B G$ space and let $\mathbf{c} \in C_{0}^{u f}(Z)$. Then, $[\mathbf{c}]=[\mathbf{0}]$ if and only if there are $r, C>0$ such that for all finite $S \subseteq Z$ we have

$$
\left|\sum_{S} \mathbf{c}\right| \leq C\left|\partial_{r}(S)\right|
$$

The main result from $\mathrm{MPR}$ that concerns us is the following.

Theorem 3.3 ([MPR, Theorem 4.15]). Let $X$ be a hyperbolic, rooted, visual graph of bounded degree. Then, $h(X)>0$ if and only if $\partial_{\infty} X$ is uniformly perfect for some visual metric.

Here $\partial_{\infty} X$ refers to the Gromov boundary of $X$ with a visual metric. While the proof is notationally involved, we summarize the main ideas. This summary will use results from [MPR]; the exact theorems and lemmas used in their proof can be found in their paper.

Summary of proof of Theorem 3.3. It suffices to prove the result for a hyperbolic approximation $X^{\prime}$ in place of $X$. The boundary at infinity, $\partial_{\infty} X=\partial_{\infty} X^{\prime}$ has strongly bounded geometry. From this and the uniformly perfect condition, one studies the combinatorics of $X^{\prime}$. By using a refinement of $X^{\prime}$, one passes to a hyperbolic approximation $X^{\prime \prime}$ for which the map $f: V_{X^{\prime \prime}} \rightarrow \mathbb{R}$ defined by $f(v)=$ $k$ for all $v \in V_{k}$ satisfies conditions which are sufficient to conclude $h(X)>0$ (particularly $\left|\nabla_{x y} f\right| \leq c_{1}$ and $\Delta f(x) \geq c_{2}>0$ for some $c_{1}, c_{2}>0$ ).

We illustrate the main ideas of how we use the above theorems with an example.

Example 3.4. Let $X=(V, E)$ be the unique infinite rooted tree where every vertex has two children (if $o$ is the root and $x, y \in V$ are distinct and connected by an edge, we say $x$ is a parent of $y$ and $y$ is a child of $x$ if $|x-o|+1=|y-o|)$. 
One can verify that $X$ satisfies a linear isoperimetric inequality (either directly or by showing $\partial_{\infty} X \simeq C_{1 / 3}$ and using Theorem 3.3. .

Define $f: V \rightarrow V$ by setting $f(o)=o$ and mapping each nonroot vertex to its parent. It is easy to check that $f$ is a quasi-isometry. We compute $f_{*}(\mathbf{V})=2 \mathbf{V}+\mathbf{o}$. Thus, $\left[f_{*}(\mathbf{V})-\mathbf{V}\right]=[\mathbf{V}+\mathbf{o}]=[\mathbf{0}]$ by Theorem 3.2 (the chain sum condition is essentially the linear isoperimetric inequality for $X)$.

Theorem 3.1 now tells us that $f$ is within bounded distance to a bilipschitz equivalence between $V$ and $V$. To see an interpretation as to why this is so, we find a chain $\mathbf{b} \in C_{1}^{b}(X)$ with $\partial \mathbf{b}=f_{*}(\mathbf{V})-\mathbf{V}$. To define the orientation for $\partial$, we note each edge $e$ consists of a parent and child vertex. We orient $e$ so that $\partial(e)$ has weights +1 on the parent and -1 on the child. Then, consider $\mathbf{b}=\sum_{E} e$. $\mathbf{A}$ quick computation shows $\partial \mathbf{b}=\mathbf{V}+\mathbf{o}=f_{*}(\mathbf{V})-\mathbf{V}$. One can interpret the chain $\mathbf{b}$ as moving the mass assigned by a bijection (corresponding to each vertex in $V$ having mass 1 , which is the chain $\mathbf{V}$ ) to that assigned by $f$ (corresponding to the chain $f_{*}(\mathbf{V})$ ). Indeed, $f$ can be constructed by starting from the identity and then "sliding" each vertex one step towards the root. In this interpretation, the weight assigned to each edge is the (signed) number of vertices "moving" along $e$.

\section{Proofs of Results}

We now combine the results in Section 3 to prove the main theorem. Recall $X$ and $Y$ are assumed to be quasi-isometric, so one of these spaces satisfies a linear isoperimetric inequality if and only if the other one does.

Proof of Theorem 1.1. Let $f: X \rightarrow Y$ be a quasi-isometry. We may assume $f: V_{X}$ $\rightarrow V_{Y}$. By assumption, $Y$ supports a linear isoperimetric inequality with constant $h=h(Y)^{-1} \in(0, \infty)$, so for any finite set $S \subseteq V_{Y}$ we have $|S| \leq h|\partial S|$. For $v \in V_{Y}$ we have $\left|\left(f_{*}\left(\mathbf{V}_{\mathbf{X}}\right)-\mathbf{V}_{\mathbf{Y}}\right)(v)\right| \leq\left|f^{-1}(v)\right|+1$. As $f$ is a quasi-isometry and $X$ has bounded degree, there is a constant $A>0$ such that for all $v \in V_{Y}$ we have $\left|f^{-1}(v)\right| \leq A$. Indeed, if $x, x^{\prime} \in V_{X}$ are such that $f(x)=f\left(x^{\prime}\right)$, then (as $f$ is a quasi-isometry, say with constants $C, D)$

$$
\left|x-x^{\prime}\right| / C-D \leq\left|f(x)-f\left(x^{\prime}\right)\right|=0
$$

and so $\left|x-x^{\prime}\right| \leq C D$. Hence, for $S \subseteq V_{Y}$ finite,

$$
\left|\sum_{S}\left(f_{*}\left(\mathbf{V}_{\mathbf{X}}\right)-\mathbf{V}_{\mathbf{Y}}\right)\right| \leq(A+1)|S| \leq(A+1) h|\partial S|=(A+1) h\left|\partial_{1} S\right|
$$

and so $\left[f_{*}\left(\mathbf{V}_{\mathbf{X}}\right)-\mathbf{V}_{\mathbf{Y}}\right]=[\mathbf{0}]$ by Theorem 3.2 with $C=(A+1) h$ and $r=1$. Thus, by Theorem $3.1 f$ is within bounded distance of a bilipschitz equivalence.

We now prove the corollaries of Theorem 1.1 and discuss the conditions in Corollary 1.2 .

Proof of Corollary 1.2. By Lemma 2.6 there exists a quasi-isometry $f: X \rightarrow Y$ that maps $V_{X}$ to $V_{Y}$. As trees are hyperbolic and essentially pseudo-regular trees are visual and have bounded degree, we may apply Theorem 3.3 to conclude that $Y$ supports a linear isoperimetric inequality. Thus, the result follows from Theorem 1.1 .

We examine the conditions of essential pseudo-regularity in Corollary [1.2, namely that $X$ and $Y$ are rooted, visual trees with bounded degree with pseudo-regular 
maximal geodesically complete subtrees. Recall that our trees being visual is important to guarantee the existence of a quasi-isometry between the trees themselves and their maximal geodesically complete subtrees. We use this property in Lemma 2.6. We also use the visual condition for the existence of a linear isoperimetric inequality from [MPR, Theorem 4.15] (here Theorem 3.3); there is a similar condition in [MPR, Theorem 3.16] which must be satisfied for a tree to support a linear isoperimetric inequality.

Pseudo-regularity guarantees that our trees branch regularly; without this condition, one tree, say $X$, would have arbitrarily long segments with no branching. If this is not the case for $Y$, then the number of vertices in balls of radius $R$ centered at any vertex grows exponentially in $R$ (see the discussion in Section 2 after the definition of pseudo-regularity; this type of bound holds uniformly for centers of balls not in the maximal geodesically complete subtree as $Y$ is visual). Suppose there is a $C$-bilipschitz map $g: V_{X} \rightarrow V_{Y}$. Consider a nonbranching segment of length $M$ in $X$. Let $x$ denote its midpoint vertex. Then, the ball $B(g(x), R)$ has at least $c^{R}$ vertices for some $c>1$ that depends only on $Y$. If $M>3 C R$, then there are at most $2 C R+1$ vertices on our nonbranching segment that could be the preimage of these $c^{R}$ vertices. As $2 C R / c^{R}<1$ for large $R$, no such $g$ can exist. Indeed, similar reasoning shows that such trees cannot even be quasi-isometric.

The bounded degree condition is similar; if the degree of $Y$ is at most $\mu<\infty$, then the number of vertices in any ball $B(y, R)$ is bounded by $c \mu^{R+1}$ for some $c>0$. Hence, if $X$ has unbounded degree and a bilipschitz equivalence $g$ exists, we would arrive at a contradiction by considering $g\left(B\left(x_{n}, 1\right)\right)$ for a sequence of vertices $x_{n}$ with strictly increasing degree in $X$.

Proof of Corollary 1.3. Note that Ahlfors regular metric spaces are uniformly perfect. By a similar construction to that in Lemma 2.6, it is known that $X$ and $Y$ are quasi-isometric (see BuS, Theorem 7.2.1] for a detailed proof or Lin16, Lemma 3.6] for a summarized proof). Theorem 3.3 shows $Y$ satisfies a linear isoperimetric inequality, so Theorem 1.1 applies.

\section{ACKNOWLEDGMENTS}

The author thanks Mario Bonk for interesting discussions on the subject matter, the anonymous referees for many helpful comments that have greatly improved the paper, and both the University of California, Los Angeles and the University of Helsinki for their hospitality.

\section{REFERENCES}

[BP] Marc Bourdon and Hervé Pajot, Cohomologie $l_{p}$ et espaces de Besov (French, with English summary), J. Reine Angew. Math. 558 (2003), 85-108, DOI 10.1515/crll.2003.043. MR 1979183

[BuS] Sergei Buyalo and Viktor Schroeder, Elements of asymptotic geometry, EMS Monographs in Mathematics, European Mathematical Society (EMS), Zürich, 2007. MR2327160

[DS97] Guy David and Stephen Semmes, Fractured fractals and broken dreams, Oxford Lecture Series in Mathematics and its Applications, vol. 7, The Clarendon Press, Oxford University Press, New York, 1997. Self-similar geometry through metric and measure. MR 1616732

[Lin16] Jeff Lindquist, Weak capacity and modulus comparability in Ahlfors regular metric spaces, Anal. Geom. Metr. Spaces 4 (2016), 399-424, DOI 10.1515/agms-2016-0019. MR.3604927

[MPM] Álvaro Martínez-Pérez and Manuel A. Morón, Uniformly continuous maps between ends of $\mathbb{R}$-trees, Math. Z. 263 (2009), no. 3, 583-606, DOI 10.1007/s00209-008-0431-5. $\operatorname{MR} 2545858$ 
[MPR] Alvaro Martínez-Pérez and José M. Rodríguez, Cheeger isoperimetric constant of Gromov hyperbolic manifolds and graphs, Commun. Contemp. Math. 20 (2018), no. 5, 1750050, 33, DOI 10.1142/S021919971750050X. MR3833900

[Pa] P. Papasoglu, Homogeneous trees are bi-Lipschitz equivalent, Geom. Dedicata 54 (1995), no. 3, 301-306, DOI 10.1007/BF01265344. MR.1326733

[Wh] Kevin Whyte, Amenability, bi-Lipschitz equivalence, and the von Neumann conjecture, Duke Math. J. 99 (1999), no. 1, 93-112, DOI 10.1215/S0012-7094-99-09904-0. MR1700742

PL 68 (Gustaf Hällströmin Katu 2B) 00014 Helsingin Yliopisto

Current address: University of Cincinnati, 2815 Commons Way, Cincinnati, Ohio 45221

Email address: Lindqujy@ucmail.uc.edu. 